\title{
MILLIMETER WAVE INTERFEROMETRY
}

\author{
D. DOWNES \\ Institut de Radio Astronomie Millimétrique \\ Grenoble, France
}

Millimeter interferometers give high-resolution (few arc sec) information on cool (100$1000 \mathrm{~K}$ ) matter, which cannot be easily studied by other techniques. The objects include regions of high extinction ( $A_{V} \sim 10-200 \mathrm{mag}$ ), planetary atmospheres, outflows from forming stars, mass loss from stellar envelopes and molecular clouds in galaxies. The advantages which $\mathrm{mm}$ arrays have over $\mathrm{cm}$ arrays are the greater number of molecular spectral lines and the larger linewidths, which allow a higher sensitivity for the same angular resolution and system performance. In the continuum mm arrays can observe solar system objects, stellar winds, compact HII regions, galaxies and quasars in thermal and synchrotron emission, and thermal radiation from dust in dense clouds. The main technical challenges are (1) sensitivity, which limits the longest usable antenna spacing, (2) small field of view, and (3) atmospheric effects, which introduce phase noise. For the next 5-10 years, the main arrays will be those of the Berkeley-Illinois-Maryland collaboration, Cal Tech, Nobeyama and IRAM. There are proposals to add more antennas to existing arrays, to build new ones, and to extend their cover to higher frequencies.

The arguments for extending the cover to higher frequencies are that the Einstein Acoefficient for molecular transitions varies as $\nu^{3}$ so, for optically thin lines, the brightness temperature, peak flux and frequency-integrated flux vary as $\nu^{2}, \nu^{4}$, and $\nu^{5}$, respectively. For optically thick lines, the peak and frequency-integrated fluxes vary as $\nu^{2}$ and $\nu^{3}$. For dust, the continuum flux density goes as $\nu^{3}$ or $\nu^{4}$, depending on grain emissivity. With these strong dependencies a high frequency array may detect many sources, even if the system is noisier than for lower frequency arrays.

Important considerations for sub-mm arrays are the greater atmospheric extinction, which limits $u, v$ plane cover with a 1-D array, even for high-Dec. sources. The observing cut-off will be $\leq 2$ air masses, so a 2 -D array is essential. The short wavelengths imply narrow fields and poor coverage of low spatial frequencies, unless the dishes are small $(\leq 6 \mathrm{~m})$. The periods of excellent sub-mm observing conditions are short, so there should be $\geq 6$ antennas, giving $\geq 15$ simultaneous baselines, for a reasonable map in one night.

There are two ratios which are lower for $\mathrm{mm}$ arrays than for $\mathrm{cm}$ arrays, maximumto-minimum baseline, and field of view-to-source size. In the first of these ratios, the minimum baseline is about the dish size, while the maximum useable baseline is limited by sensitivity. Unlike most $\mathrm{cm}$ arrays, $\mathrm{mm}$ arrays have had smaller and fewer antennas, higher system temperatures, more atmospheric problems, and weaker calibrator sources. For mm arrays until now, the minimum detectable brightness temperature in the synthesised beam is a few $\mathrm{K}$ at a $100 \mathrm{~m}$ baseline. However, the temperatures of thermal line sources are also 
a few $\mathrm{K}$, so it has been hard to get useful signal-to-noise ratios at baselines $>100 \mathrm{~m}$.

The synthesised beams for typical baselines used at $3 \mathrm{~mm}$ are $4^{\prime \prime} 11^{\prime \prime}$, close to the sizes for which information is missing from the maps because of the minimum baseline. At $2.6 \mathrm{~mm}$, a minimum spacing of $25 \mathrm{~m}$ reduces the visibility of $9^{\prime \prime}$ structures by half, and structures $>20^{\prime \prime}$ to zero. In principle, missing zero-spacing information can be recovered from maps by single dishes like the Onsala $20 \mathrm{~m}$, IRAM $30 \mathrm{~m}$ and Nobeyama $45 \mathrm{~m}$ telescopes. Other ways to get the short-spacing information are scanning the antennas during the synthesis and sub-aperture illumination of the dishes.

The second ratio which is less favourable for $\mathrm{mm}$ arrays than for $\mathrm{cm}$ arrays, is the field of view ( $\sim 1^{\prime}$ at $3 \mathrm{~mm}$ ) relative to the size of typical sources, namely several arc min for molecular clouds and nearby galaxies. To overcome this problem, a mosaic of adjacent fields could be observed by multi-beaming of several fields simultaneously, or by observing the fields sequentially, with only one receiver per antenna. Another possibility might be to combine data from different arrays, e.g., the Hat Creek array could synthesise a large field at short baselines, and the IRAM array could observe the field hot spots at longer baselines.

Another effect with stronger consequences for mm arrays than for $\mathrm{cm}$ arrays, because of the smaller primary beams, is anomalous refraction. In these events sources appear to move by $\leq 20^{\prime \prime}$ for $\leq 30 \mathrm{sec}$ of time. Anomalous refraction is caused by variations in the "wet" component of the refractive index and in $\mathrm{mm}$ and sub-mm interferometry it will increase phase noise, broadening the synthesized beam, and will reduce fringe amplitude as sources move out of the narrow primary beams.

As the number of antennas in $\mathrm{mm}$ arrays increases, it may be possible to use optical interferometry methods to overcome atmospheric phase errors, such as the bi-spectrum method in which triple products of the complex visibility are formed on short time scales, then ensemble averaged to improve sensitivity, and then used to derive phases. This method may improve phase closure or self-calibration methods by 3-10 times in sensitivity.

Table 1 lists current and proposed $\mathrm{mm}$ arrays, representing different approaches to solving these problems. Probably both types of array will be useful, those with wide field of view, and those with high sensitivity on longer baselines. Given the active interest in enlarging existing arrays, in constructing new and more powerful ones, and the rewards to be obtained at higher frequencies, $\mathrm{mm}$ interferometry obviously has a very promising future.

\section{TABLE I Existing and proposed millimeter and submillimeter arrays}

\begin{tabular}{lcccc}
\hline Array/Status & $\begin{array}{c}\text { Number of } \\
\text { Antennas }\end{array}$ & $\begin{array}{c}\text { Instantaneous } \\
\text { Area }=n \pi D^{2} / 4 \\
\left(\mathrm{~m}^{2}\right)\end{array}$ & $\begin{array}{c}\text { Shortest } \\
\text { Usable } \\
(\mathrm{mm})\end{array}$ & $\begin{array}{c}\text { Relative } \\
\text { Speed for full } \\
\text { synthesis }\end{array}$ \\
\hline Hat Creek (in operation) & $3 \times 6 \mathrm{~m}$ & 85 & 1.3 & 1.0 \\
Hat Creek (funded) & $6 \times 6 \mathrm{~m}$ & 170 & 1.3 & 5.0 \\
Owens Valley (in operation) & $3 \times 10.4 \mathrm{~m}$ & 255 & 1.3 & 1.0 \\
Nobeyama (in operation) & $5 \times 10 \mathrm{~m}$ & 393 & 1.3 & 5.0 \\
Nobeyama (future) & $+45 \mathrm{~m}$ & 1983 & 1.3 & 5.0 \\
Plateau de Bure (construction) & $4 \times 15 \mathrm{~m}$ & 530 & 0.8 & 1.0 \\
Plateau de Bure (funded) & $4 \times 15 \mathrm{~m}$ & 707 & 2.6 & 2.0 \\
Australia T'scope (later phase) & $5 \times 15 \mathrm{~m}$ & 884 & 2.6 & 3.3 \\
Smithsonian (proposed) & $6 \times 6 \mathrm{~m}$ & 170 & 0.35 & 5.0 \\
NRAO (current idea) & $40 \times 7.5 \mathrm{~m}$ & 1740 & $\sim 1$. & 63.3 \\
\hline
\end{tabular}

